\title{
Perspective
}

PERSPECTIVE Actualité en histoire de l'art

$2 \mid 2020$

Danser

\section{Saisir la danse dans les images. Étude sur l'intentionnalité chorégraphique des arts visuels du XVII ${ }^{\mathrm{e}}$ siècle}

Capturing Dance in Images: A Study of Choreographic Intentionality in 17thCentury Visual Arts

Den Tanz in Bilder fassen. Studie zur choreografischen Intentionalität der

bildenden Kunst im 17. Jahrhundert

Cogliere la danza nelle immagini. Studio sull'intenzionalità coreografica delle

arti visive del XVII secolo

Atrapar la danza en las imágenes. Estudio sobre la intencionalidad coreográfica

de las artes visuales del siglo XVII

\section{Bianca Maurmayr}

\section{OpenEdition}

Journals

Édition électronique

URL : https://journals.openedition.org/perspective/20826

DOI : 10.4000/perspective.20826

ISSN : 2269-7721

Éditeur

Institut national d'histoire de l'art

Édition imprimée

Date de publication : 30 décembre 2020

Pagination : 155-166

ISBN : 978-2-917902-90-5

ISSN : $1777-7852$

Référence électronique

Bianca Maurmayr, «Saisir la danse dans les images. Étude sur l'intentionnalité chorégraphique des arts visuels du xvII e siècle », Perspective [En ligne], 2 | 2020, mis en ligne le 30 juin 2021, consulté le 30 juillet 2022. URL : http://journals.openedition.org/perspective/20826 ; DOI : https://doi.org/10.4000/ perspective. 20826 


\title{
Saisir la danse dans les images Étude sur l'intentionnalité chorégraphique des arts visuels du XVII ${ }^{e}$ siècle
}

\author{
Bianca Maurmayr
}

"Les mouvements complexes de la danse ne sauraient être communiqués par une simple représentation ${ }^{1}$. " C'est ainsi que Sydney Anglo résume un écueil méthodologique majeur pour la recherche en art dans la lecture des images : un tableau, une gravure ou une estampe ne peuvent que reproduire un mouvement isolé, décontextualisé des actions qui le précèdent ou le suivent immédiatement, ce qui porte l'observateur d'aujourd'hui à avancer un grand nombre d'hypothèses sur les mouvements que ces images peuvent bien représenter. Il se peut que l'image ne témoigne pas d'un style, d'un pas ou d'un geste précis $^{2}$ mais évoque simplement l'idée de mouvement. À moins que l'auteur de l'image ne fournisse une série de parataxes et/ou une description du geste qui permettent de saisir la réalisation du mouvement, étape par étape, l'immobilité de la source iconographique risque de l'emporter sur la compréhension du mouvement représenté.

Cependant, nous sommes d'accord avec Judy van Zile lorsqu'elle affirme que les divergences entre une supposée réalité historique et les images qui s'y réfèrent ne peuvent invalider l'utilité de ces dernières ${ }^{3}$. Dans cet article, il s'agira précisément de comprendre comment la recherche en danse actuelle peut approcher les sources iconographiques historiques, en tenant compte des caractéristiques propres aux images pour représenter le mouvement - le style de l'auteur, la tradition graphique, le support de l'image, le contexte de production. Afin de saisir le motus du mouvement, le chercheur ou la chercheuse en danse devra faire appel à son intelligence kinésique, faculté cognitive qui permet de sémantiser et de comprendre les mouvements et postures grâce à l'expérience du mouvement et, comme nous le verrons, grâce aussi au savoir sur les pratiques corporelles qu'il ou elle souhaite creuser.

Afin de montrer ce que la recherche peut tirer de ce procédé, nous nous appuyons ici sur l'étude des Balletti d'Invenzione nella Finta Pazza ${ }^{4}$, suite d'estampes publiée à Paris aux alentours de 1645 par le compositeur de ballets Giovanni Battista Balbi, confrontée aux traités d'époque, à quelques images de ballet des peintres et graveurs français du XVII ${ }^{\mathrm{e}}$ siècle tels que Daniel Rabel (1626) ou Jean Ir Berain (vers 1680), ou encore aux gravures de Johann Puschner pour le manuel de Gregorio Lambranzi Nuova e Curiosa Scuola de Balli Theatrali (1716), dans une perspective synchronique et diachronique. 
Il s'agit de montrer que l'analyse kinésique, ancrée dans une dimension corporelle et expérientielle, de ce document de la danse permet d'identifier et d'étudier certains principes moteurs de la danse du XVII ${ }^{e}$ siècle. Nous insistons donc sur la réception des images depuis la perspective académique actuelle, pour construire un savoir partagé et historiquement informé sur les pratiques corporelles et dansées de l'époque moderne.

\section{L'intentionnalité chorégraphique, ou ce que l'image nous transmet de la danse}

Dans le contexte de son enquête sur la relation entre art et spectacle vivant, Sara Mamone désigne la valeur épistémologique de l'image en employant l'expression d' « intentionnalité spectaculaire du monument / document ${ }^{5}$ " iconographique. Celle-ci dépend du degré de connaissance, directe ou indirecte, que l'auteur de l'image a de l'événement représenté, et de sa volonté de réaliser un document qui témoigne de l'acte performatif en soi ou, au contraire, du degré de "non-préméditation " spectaculaire. La chercheuse propose même d'établir « une sorte d'échelle de l'intentionnalité spectaculaire, c'est-à-dire de déterminer cas par cas à quel point le témoin a voulu transmettre un document de spectacle qui soit perçu immédiatement comme tel, ou combien cette intentionnalité se dilue et se nuance dans d'autres genres d'intentionnalités ${ }^{6} »$. Pour illustrer ce propos théorique et l'appliquer à la danse, nous souhaitons étudier le cas du volume Balletti d'Invenzione nella Finta Pazza, décrivant à travers un ensemble d'estampes trois ballets de Giovanni Battista Balbi destinés à servir d'intermèdes pour la première représentation publique en France d'un opéra, ouvrage dont on peut dire que l' « intentionnalité spectaculaire " est relativement explicite. Relié en maroquin noir et portant le monogramme de la reine Anne d'Autriche en signe de dédicace, il est publié par le Ballarino Veneziano celebre, Balbi, appelé à Paris en 1645 à la cour du jeune Louis XIV pour compléter la troupe des Comédiens Italiens. Sa présence, associée à celle du décorateur, machiniste, mathématicien et ingénieur expert Giacomo Torelli et d'autres artisti di giro, est liée à la réalisation de La Finta Pazza, chef-d'œuvre de la première phase du théâtre public vénitien, représenté à Paris, au théâtre du Petit-Bourbon, le 14 décembre $1645^{7}$. Le volume rend précisément compte des entrées composées par Balbi à cette occasion; il s'agit, dans l'ordre, du "Ballet des Cinges \& des Ours", avec quatre eunuques turcs balayant et arrosant le sol, du « Ballet des Autruches " qui se rafraîchissent à un abreuvoir, et du "Ballet des Indiens $\delta$ des Perroquets " avec parasols et bâtons. Pour chacune des entrées, on trouve un texte qui la contextualise et six planches numérotées, pour un total de dix-huit gravures. Il s'agit donc de la représentation intentionnelle d'un acte performatif, exécutée par deux artistes liés au milieu du spectacle : Stefano Della Bella, inventeur des fêtes florentines, chargé des dessins préparatoires, et Valerio Spada, calligraphe et graveur toscan, chargé de la gravure du volume et auteur de la page de titre - comme le suggère la mention "Valerio Spada fecit ${ }^{8}$ ". L'épître dédicatoire de Balbi à la Reine Mère, manuscrite, l'avis au lecteur qui explique les difficultés de l'entreprise ainsi que les rehauts d'or et d'argent des planches de la version conservée dans les fonds de la bibliothèque de l'Institut national $\mathrm{d}^{\prime}$ histoire de l'art ${ }^{9}$, permettent d'identifier le volume à un livre de prestige ${ }^{10}$.

Bien qu'il semble clair que ce document ait été produit pour témoigner d'un spectacle, il est moins sûr que nous puissions lui reconnaître une "intentionnalité chorégraphique ". Créée sur la base de celle d' "intentionnalité spectaculaire " forgée par Mamone, nous employons cette expression pour interroger la capacité du document iconographique et des images qu'il contient à témoigner de la composition dansée en elle-même - ce qui implique une tentative de notre part d'enquêter sur la connaissance que Della Bella avait 
de la danse et sur la perception immédiate par laquelle on peut reconnaître un mouvement de danse sous ses traits stylistiques. Il nous semble imprudent de croire que Della Bella aurait reproduit fidèlement tous les mouvements exécutés sur scène par les danseurs de Balbi : si les poses - comme nous le verrons - peuvent être lues à l'aune des pratiques dansées de l'époque, elles se réduisent à des constructions gravitaires peu variées. Les figures sont en effet représentées dans des positions ouvertes ou en suspension sur un pied, l'autre jambe en l'air et éventuellement croisée avec la première, ce qui suggère le principe d'élévation du saut (fig. 1). Par ailleurs, d'un point de vue formel, elles ont peu de relief, ce qui rend leur interprétation difficile.

Au premier abord, il semblerait que Della Bella ait préféré ici, à la reproduction du geste chorégraphié, celle du "trait suggestif du mouvement ${ }^{11}$ ", en le formalisant dans une figure suspendue en l'air, un des deux pieds ne touchant pas le sol pour évoquer une marche ornementée. Il transmet cette idée de mouvement par la flottaison des corps, l'animation des plumes des chapeaux, et par la suspension des figures prises dans le "faire ", suivant en cela la tradition iconographique du XVI ${ }^{e}$ siècle ${ }^{12}$. D'autre part, nous ne pouvons pas ignorer le style de l'auteur, qui se définit par sa légèreté, sa flexibilité, voire par " la souplesse de son écriture ${ }^{13}$ "; si d'élan il s'agit, il pourrait alors être conditionné par un style aérien. Il est en revanche intéressant de souligner le choix de laisser la danse se dérouler sur la page, et d'une page à l'autre, en dehors de son contexte de production, autrement dit la mise en scène et la scénographie de La Finta $P_{\text {Pazza }}{ }^{14}$. Si, en d'autres occasions, notamment dans Le Nozze degli dei (Florence, 1637), Della Bella avait privilégié une vue topographique, ici, la perspective adoptée semble être celle du bénéficiaire principal de l'œuvre, le roi lui-même, afin de lui rappeler ce que " fut son premier grand bonheur artistique ${ }^{15}$ ", dérivé de ces danses exotiques au sujet animal et paramilitaire.

\section{Procédé méthodologique expérimental pour la lecture des images}

Afin de dépasser l'apparente " non-préméditation chorégraphique ${ }^{16}$ " de cette source, il est possible, pour approcher le mouvement dansé, de faire appel à la "simulation perceptive " de Guillemette Bolens. Dans l'introduction à son ouvrage Le Style des gestes, la chercheuse nous présente ce processus cognitif à travers l'analyse kinésique d'une image, et plus particulièrement du portrait d'Auguste-Gabriel Godefroy par Jean-Baptiste Chardin, connu comme L'Enfant au toton (Salon de 1738, aujourd'hui conservé au musée du Louvre), qui, les yeux fixés sur son jouet, vient de lancer la toupie et s'attarde à la voir tourner :

La compréhension de l'image se fait [...] par l'inférence d'une relation entre la toupie et la main, fondée sur la position de celle-ci et sur l'interprétation du regard de l'enfant. Le spectateur projette sur ce regard une intention, laquelle est au passé (l'enfant a voulu faire tourner la toupie et a réalisé son intention). Et il a fait également l'hypothèse anticipatrice que l'enfant anticipe lui-même le ralentissement et la chute du jouet, avec l'intention supplémentaire de renouveler son geste avec telle nuance dynamique dans la mise en acte de l'impulsion motrice qu'il impartira de nouveau à la toupie. [...] Sans l'intelligence kinésique de celui qui regarde, la toupie ne tourne pas et l'image reste lettre morte ${ }^{17}$.

La lecture du geste se construit ainsi entre l'œuvre picturale et la perception que l'observateur en fait : ce dernier comprend la relation instaurée entre les différents objets mouvants (la main d'Auguste-Gabriel, mais aussi un pied, une jambe, la tête, la colonne vertébrale) 
et mus (la toupie, ou, dans les Balletti, un balai, un arrosoir, un tambour) et construit sa propre interprétation incorporée du geste représenté, instruit du savoir kinesthésique approprié - dans notre cas, la pratique dansée du XVII siècle. C'est donc grâce à sa faculté d'exploiter les simulations perceptives, ou à son "intelligence kinésique ", que l'image se libère idéalement de sa condition statique et prend vie.

Ce procédé de lecture des images peut être associé à la méthodologie de la recherche en danse qui fait la part belle à l'incorporation, à l'expérimentation et à l'activation de la source. Les avertissements, les règles ou les manières des traités des XVI ${ }^{\mathrm{e}}$ et XVII ${ }^{\mathrm{e}}$ siècles ne peuvent en effet être saisis que par la mise en corps de leur contenu ; on peut en dire autant de la relation entre les descriptions des pas ou les systèmes de notation qui encodent le geste et les images qui les accompagnent. La lecture devient dès lors une action cognitive multiple et dynamique, qui associe aux réflexes des yeux et de l'appareil phonatoire l'activation des muscles - de la jambe dont on explique une flexion, des bras dont on décrit une position, des appuis dont on suggère un transfert pour mieux gérer le poids. Ainsi, la simulation perceptive et l'expérimentation permettent de re-somatiser le texte, le trait du peintre ou les signes de la notation, afin d'en faire la source possible d'une série d'hypothèses qui émane du corps. Pour le dire avec les mots de Susan Leigh Foster :

Oui, la production de l'histoire est un effort physique. [...] Une fois que le corps de l'historien·ne reconnaît la valeur et le sens de la kinesthésie, il ne peut pas dés-animer l'action physique des corps passés qu'il a commencé à percevoir ${ }^{18}$.

\section{Lire entre les lignes : analyse biomécanique et graphique à l'œuvre}

Une telle lecture de la source iconographique qui procède par simulation et par incorporation depuis la réception de l'observateur actuel, lorsqu'elle est instruite d'un savoir-faire dansé, nous permet également de relever et d'étudier les effets produits par les possibles incongruences entre les choix graphiques de l'artiste et l'expérimentation du geste représenté.

Dans le cas des Balletti, le plan perspectif des images est très ample mais pas suffisamment profond, ce qui a pour effet d'écraser les figures et de leur conférer des qualités corporelles contre-intuitives. La sensation kinesthésique d'orientation des pieds dans les deux diagonales avant du corps et d'en-dehors semble, précisément, être amplifiée par les effets de tassement (sur le plan vertical) et d'élargissement (sur le plan horizontal) causés par ce choix perspectif, qui entraîne une dilatation des figures en largeur et accentue la rotation externe des articulations ${ }^{19}$. En même temps, il faut rappeler ici que la rotation en-dehors des pieds était déjà mentionnée par Cesare Negri dans Le Gratie d'amore (1602) et par l'auteur anonyme de l'Instruction (1610), puis par Ercole Santucci da Perugia dans Mastro da ballo (1614) et par François de Lauze dans Apologie (1623) ; elle était dès lors inscrite dans les savoir-faire chorégraphiques de l'époque de Balbi. De plus, d'un point de vue biomécanique, agrandir la surface de la base d'appui en orientant les pointes des pieds vers les deux diagonales avant garantit une meilleure

1. Stefano Della Bella et Valerio Spada, «Ballet des Cinges \& des Ours [sic] », première entrée, troisième planche, dans Balletti d'Invenzione nella Finta Pazza di Giovanbatta Balbi, vers 1645, Paris, bibliothèque de I'INHA - collections Jacques Doucet.

2. Stefano Della Bella et Valerio Spada, «Ballet des Autruches ", deuxième entrée, deuxième planche, dans Balletti d'Invenzione nella Finta Pazza di Giovanbatta Balbi, vers 1645, Paris, bibliothèque de I'INHA - collections Jacques Doucet. 

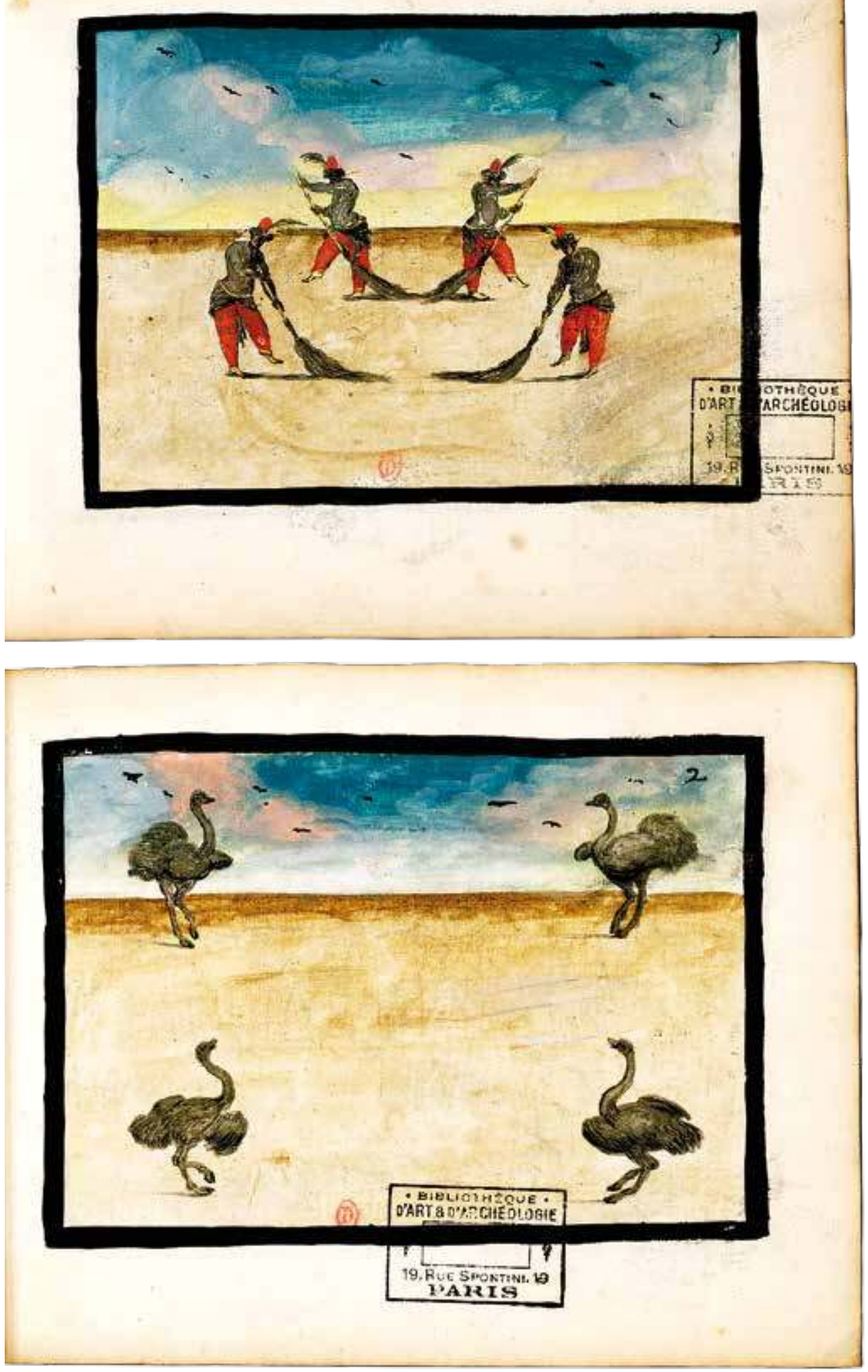


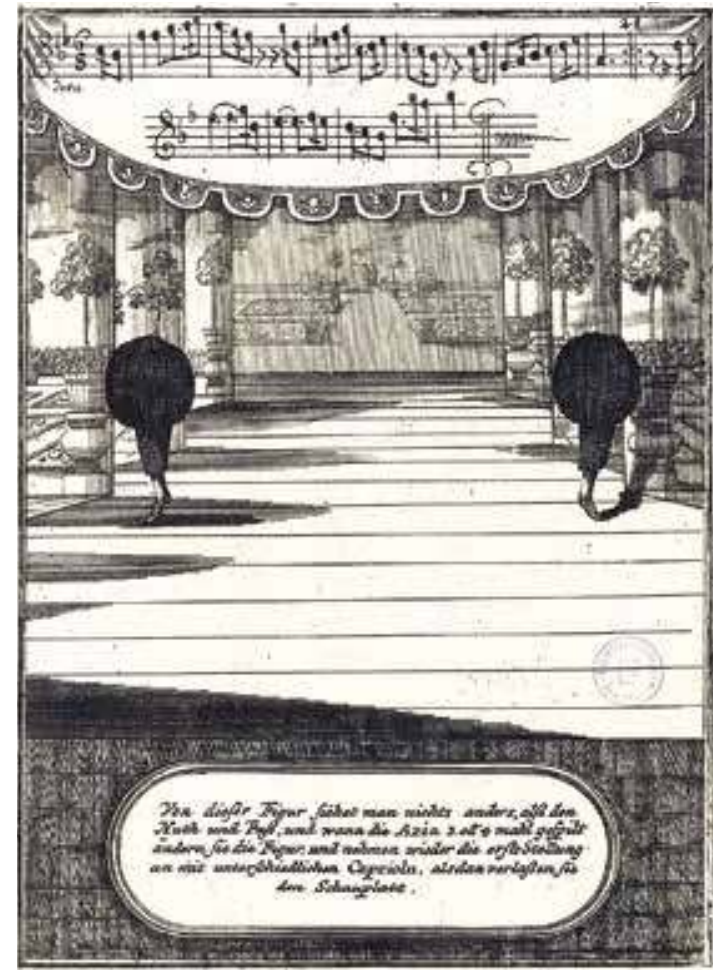

3. Johann Georg Puschner, entrée où « I'on ne voit rien d'autre que le chapeau et le pied ", dans Gregorio Lambranzi, Neue und curieuse theatrialische Tantz Schul : deliciae theatrales, Nuremberg, verlegts Johan Jacob Wolrab, 1716, n. p., Madrid, Biblioteca Nacional de España. stabilité du corps et facilite le contrôle de la posture orthostatique. Pour des danseurs professionnels comme ceux engagés dans La Finta Pazza, cela signifie pouvoir effectuer les croisements des pieds, les battus ou les ronds de jambe de manière plus aisée et organique. Conditionnement graphique et expérimentation corporelle ne s'éliminent donc pas mutuellement ici.

Un questionnement semblable surgit à l'observation des planches de la deuxième entrée, mettant en scène une danse d'autruches (fig. 2). Constatant que, sur le dessin, l'orientation vers l'arrière des genoux de l'autruche était respectée, Philippe Beaussant s'est demandé comment les danseurs devaient endosser le costume, sans pouvoir "guère imaginer autre chose que des enfants $[\ldots]$ marchant et dansant à l'envers pour avoir les genoux vers l'arrière, comme on nous le montre ${ }^{20} "$. Selon une reconstitution du costume de l'autruche par Jane Stein pour une recréation commissionnée à Catherine Turocy en 2014 ${ }^{21}$, ce choix s'est avéré peu fonctionnel. Pour rentrer dans le costume et incorporer l'animal, le danseur doit réorganiser son propre équilibre postural et fléchir la colonne, opération qui entraîne un déplacement segmentaire sur le plan horizontal : lorsque l'on se penche en avant, la ceinture pelvienne recule. Ce processus de translation en sens opposé de la cage thoracique vers l'avant, et du bassin vers l'arrière, nécessairement amplifié par le costume, ne permet de garder la projection

du centre de gravité dans la base d'appui, et de conserver l'équilibre, que si l'on se tient dans le même sens que l'animal représenté. Si le costume était endossé avec le devant derrière, le poids de la tête et du cou de l'animal entraînerait le corps en arrière et provoquerait la chute. La confrontation des analyses graphique et biomécanique de l'image semble ici attester le choix de Della Bella de l'imitation de la morphologie de l'animal, et non de celle des interprètes en train de danser, ce qui répond à la fonction du volume : appartenir à la collection de la famille royale et, donc, satisfaire le regard diverti que le jeune roi avait pu porter sur ces danses.

\section{Lecture kinésique des images de Balbi, à l'aune de l'intertextualité}

L'approche intertextuelle ${ }^{22}$ vient renforcer le processus méthodologique d'incorporation et d'expérimentation de la source par simulation perceptive : les traités d'époque fournissent un appui à l'expérience kinesthésique du chercheur, qui est dès lors en position de formuler des hypothèses de lecture du geste historiquement informées, tandis que le dialogue avec d'autres images aide à identifier des conventions théâtrales et chorégraphiques liées à la représentation de certains personnages. 
Dans la composition de l'entrée des autruches que nous venons d'évoquer, la contrainte du costume joue un rôle majeur, étant entendu aussi que la seule partie du corps des danseurs laissée libre sont les jambes. Inventeur des habits de ses propres entrées, Balbi avait dû imaginer des stratégies créatives qui transformaient cette entrave en source d'expérimentation et de renouveau chorégraphique ${ }^{23}$; et pourtant, ces stratégies ne sont pas lisibles dans les gravures de Stefano Della Bella et Valerio Spada, dont la composition paraît se réduire à la construction de figures géométriques dans l'espace. C'est le dessin de Puschner, d'une entrée comique où " l'on ne voit rien d'autre que le chapeau et le pied » (fig. 3), qui nous donne une clé de lecture possible de ces démarches : représentés dans leur tentative de se libérer du poids du costume lourd et bombé, les danseurs se livrent à des " cabrioles", sauts où les jambes battent l'une contre l'autre ; néanmoins, la forme dans laquelle ils sont emprisonnés les rabaisse inévitablement au sol. Nous laissant guider par cet exemple, nous pouvons avancer l'hypothèse que Balbi s'est plutôt fait caricaturiste des autruches, en faisant deviner les mouvements qui les caractérisent - une marche enjambée et la tentative de l'envol - et en en rendant visible l'échec par des moyens techniques ardus - des sauts battus en l'air qui transmettent un désir d'élan mais qui échouent dans l'enchevêtrement de leurs pattes.

En ce qui concerne la première entrée, procédons d'abord à l'analyse graphique et biomécanique des planches avant la confrontation intertextuelle - autrement dit, la comparaison avec un corpus d'images et de documents issu de contextes comparables. À l'exception de la première planche, les Turcs sont portraiturés dans l'action de déplacement avec une jambe en l'air, la plante du pied tournée vers le sol, prêts à poser le pied au

4. Atelier de Daniel Rabel, "Seconde entrée du grand Seigneur / Dansant avecque ses Suivans : dessin ", costumes pour la deuxième partie du ballet Grand Bal de la douairière de Billebahaut, intitulée "Ballets de I'Asie ", 1626, Paris, Bibliothèque nationale de France, département Estampes et Photographie.

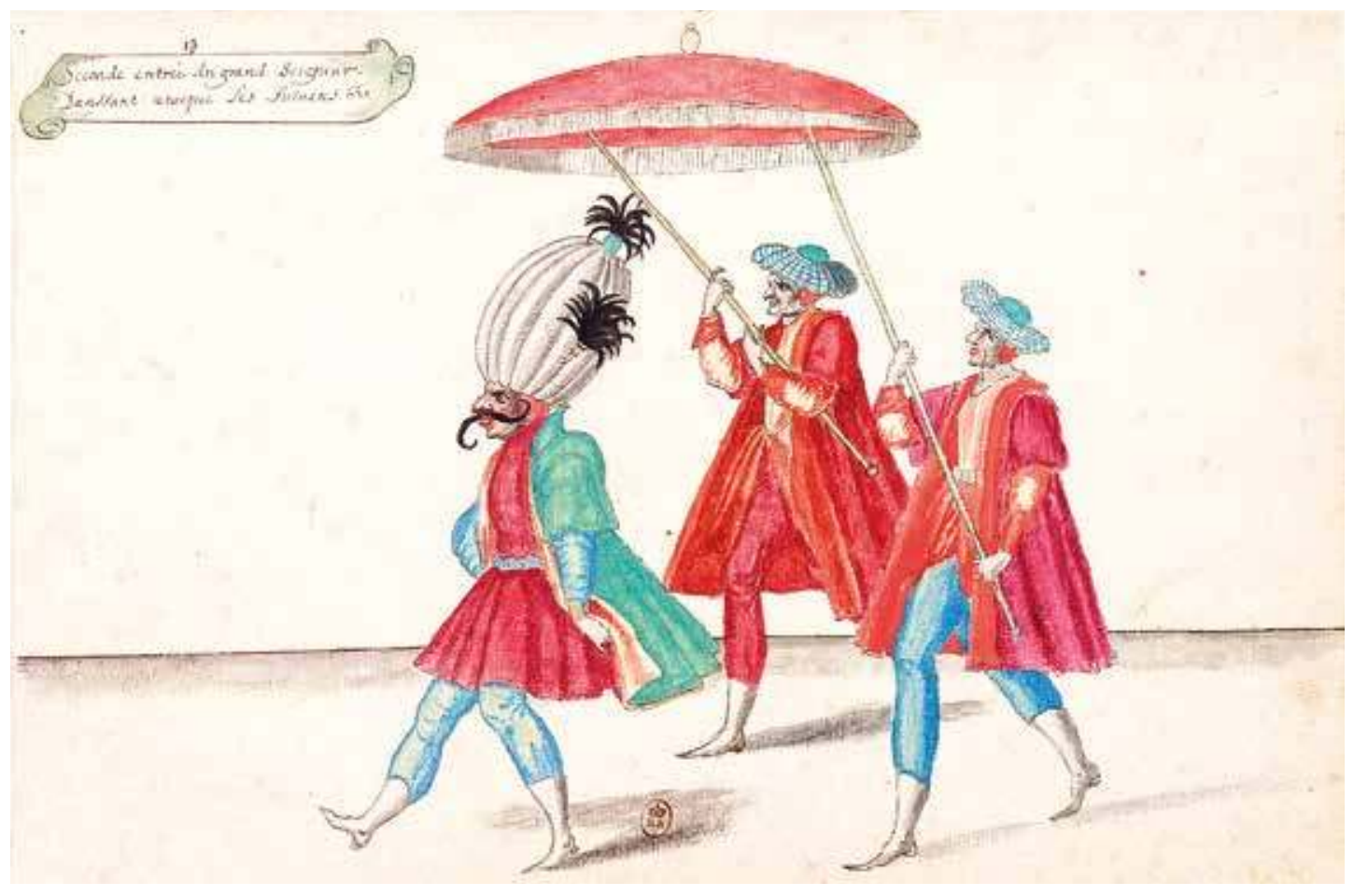


sol. Le peu de profondeur des images et le style aérien de Della Bella empêchent de saisir précisément le rapport à la gravité des danseurs. Si nous observons la figure à l'avant-plan gauche de la troisième planche (fig. 1), nous sommes portés à croire en une résistance forte à la gravité : on dirait que le danseur est en train de dérouler son pied pour se lancer dans un saut, car il a le talon légèrement soulevé du sol, la taille bien marquée et le bassin suspendu vers le haut - ce qui allonge sa ligne musculaire arrière superficielle et amincit sa figure. Les figures des trois autres danseurs ne produisent pas la même perception motrice : ils semblent plutôt tassés vers le sol. Cette différence de traitement graphique des figures est perceptible dans une division de la planche par plans : ni les centres de gravité, ni les épaules des deux personnages à l'avant-scène ne sont alignés, faisant du danseur côté cour une figure considérablement plus petite et corpulente que son partenaire côté jardin ${ }^{24}$. C'est précisément parce qu'elle ouvre deux hypothèses motrices possibles pour le personnage du Turc que cette planche nous paraît intéressante à analyser.

L'organisation gravitaire lourde et proche du sol rappelle celle conférée par Daniel Rabel au Grand Turc du Ballet du Grand Bal de la Douairière de Billebahaut (fig. 4). Le centre de gravité y est bas et déséquilibré vers l'arrière par rapport à l'appui de la jambe de terre - perception renforcée par le volume que prennent les haut-de-chausses et le bas du manteau, ridiculisant la figure. Le genou de la jambe de terre

5. Martin Engelbrecht, " Masque en Habit dansante à l'Opéra », dans Habillements de l'Opéra : cinquante-trois planches, volume relié de gravures, réalisé par les Menus-Plaisirs du roi, vers 1750, p. 43, Paris, Bibliothèque nationale de France, bibliothèque-musée de l'Opéra.

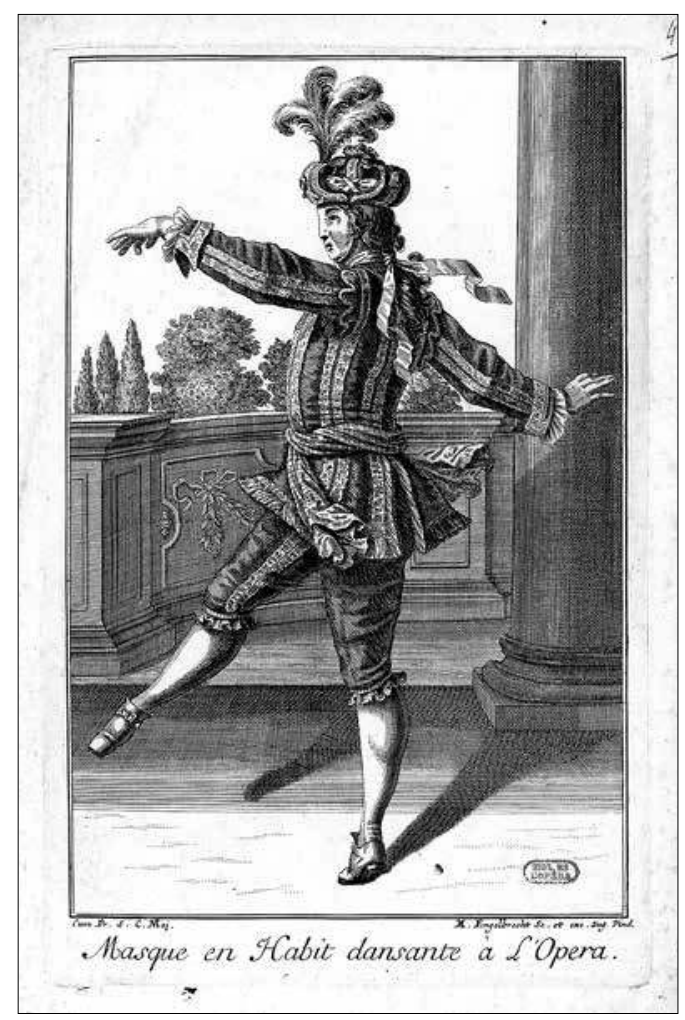
plié, et le pied en l'air en flexion, soutiennent à leur tour l'hypothèse d'une corpulence lourde et d'une marche proche du sol. On trouve une organisation semblable du corps en mouvement dans la représentation par Jean Berain le Vieux de Deschars en Polichinel au Divertissement de Villeneuve Saint-Georges ${ }^{25}$ (1680), qui a ici le pied posé au sol : la marche à pieds plats et la surcharge du poids du danseur par le costume déclenchent un effet comique.

Quant à l'organisation gravitaire davantage soutenue, indiquant peut-être même un effet sautillé, elle ne peut être écartée de manière arbitraire : dans la danse manuscrite " Türkish Dance » (1725), attribuée à Anthony L'Abbé et admirablement étudiée par Dóra Kiss ${ }^{26}$, les danseurs, habillés en Turcs, démarrent la danse par une marche à pieds plats, puis la terminent par un déplacement latéral vers le côté cour qui s'arrête dans un équilibre précaire, sur demi-pointe, une jambe levée croisée devant eux. Cette organisation rappelle non seulement la planche de Balbi, mais encore plus l'une des gravures du manuel de Lambranzi (II.38), où les danseurs, représentant des personnages de Turcs, sont dits être en train d'exécuter des "ballonnés ". Notons que nous retrouvons cette pose dans le « Masque en Habit dansante à l'Opéra » de Martin Engelbrecht (fig. 5), peut-être un Oriental ou un Mezetin anoblii ${ }^{27} ; \mathrm{si}$, au premier abord, on pourrait croire à une convention graphique pour rendre la danse, l'attention portée 


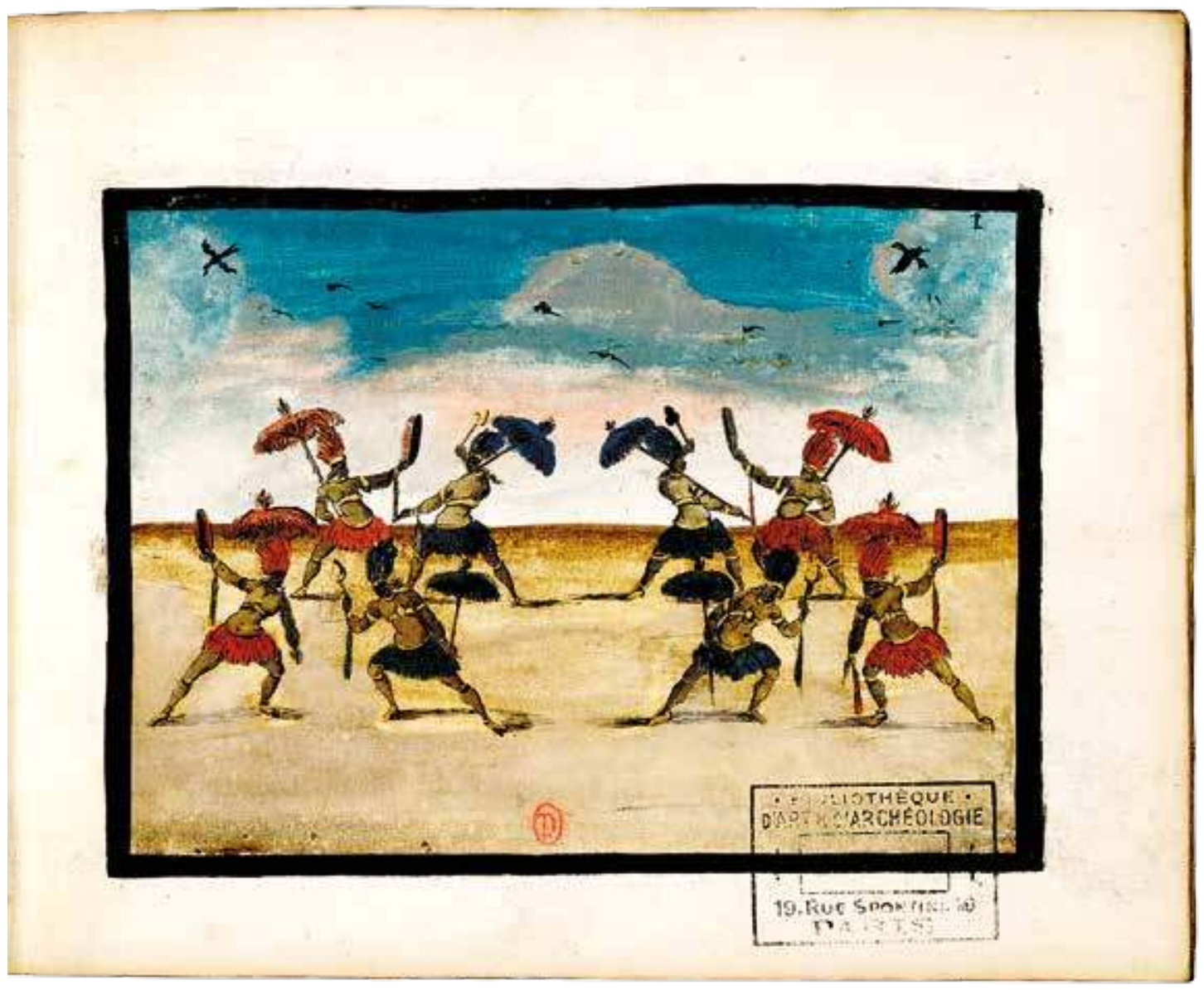

sur l'effort musculaire du mollet lors de l'élévation témoigne d'une observation directe par l'auteur de la corporéité dansante.

Sur la base de ces résonances, nous pouvons émettre l'hypothèse d'un effet comique recher6. Stefano Della Bella et Valerio Spada, «Ballet des Indiens \& des Perroquets ", troisième entrée, première planche, dans Balletti d'Invenzione nella Finta Pazza di Giovanbatta Balbi, vers 1645, Paris, bibliothèque de I'INHA - collections Jacques Doucet. ché par Balbi, produit par l'alternance entre les tentatives d'élévation et les redescentes des Turcs au sol, déclenchant le rire du public. L'usage, à des fins chorégraphiques, d'outils de métier tels le balai et l'arrosoir, vient renforcer cette impression de ridicule et d'inadéquation. A contrario, l'utilisation des bâtons dans la troisième entrée des Indiens, associée à l'attitude corporelle virile ${ }^{28}$ des danseurs (fig. 6), relie cette danse aux gestes mesurés des gravures de Jacob de Gheyn II pour le Maniement d'armes, d'arquebuses, mousquets et piques (1608) ou d'un carnet de croquis d'un maître de danse bruxellois conservé à la bibliothèque royale de Stockholm (Cod. S. Holm 253), récemment étudié par Jennifer Nevile ${ }^{29}$. Bâtons et parasols pouvaient, dès lors, tourbillonner en l'air et être passés sur le cou, l'épaule ou le dos de la main, créant un effet de maestria. De même, l'image du Balletto finale con i bastoni de Giovanni Tommaso Borgonio (fig. 7), et les artige Erfindungen ( inventions astucieuses ") proposées par Lambranzi dans sa planche II.18, portent à penser que les danseurs pouvaient aussi former des figures avec les bâtons (couloirs, carrés, moulins) afin d'agrémenter l'espace de la danse. 


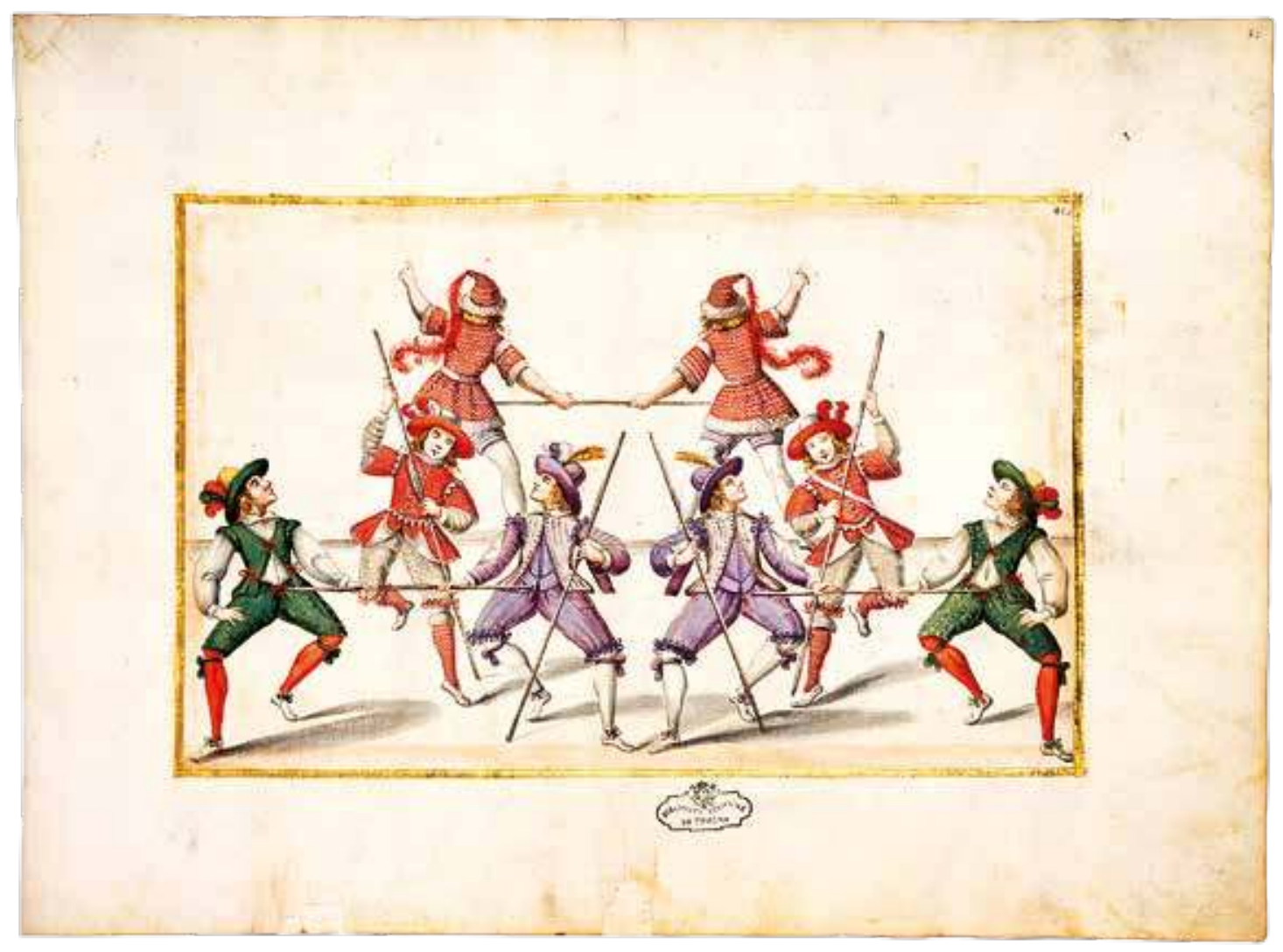

7. Giovanni Tommaso Borgonio, Filippo d'Aglié, Dono del Re delle Alpi à Madama Reale, 1645, Turin, Biblioteca nazionale universitaria, fo 38 .
Ainsi, l'analyse intertextuelle et expérimentale nous porte à avancer des hypothèses kinésiques pour l'utilisation des bras : d'une part, l'entrée des eunuques nous suggère que les danseurs grotesques pouvaient imiter un travail mécanique, à la différence des gestes des danseurs nobles qui devaient privilégier l'ornementation ; d'autre part, l'entrée des Indiens nous renseigne en creux sur la forme

des moresques et danses militaires, dont l'abbattimento con bastoni ( combat avec des bâtons "), des XVI ${ }^{\mathrm{e}}$ et XVII ${ }^{\mathrm{e}}$ siècles ${ }^{30}$.

Pour conclure, la lecture kinésique des planches des Balletti nous aide à redonner corps et mouvement aux images préparées par Della Bella et gravées par Spada, au travers d'un procédé comparatif et diachronique, transhistorique. Bien que non exhaustive, cette étude de cas démontre que la compréhension du geste représenté dans une image se trouve à la confluence de la reproduction du tonus musculaire et de la construction gravitaire observés, de l'habileté technique et du style de l'auteur, souvent déterminants, de la nature matérielle du document iconographique, ainsi que de la faculté cognitive de l'observateur à conférer une intention motrice au geste. Tenant alors compte des conditions de production et de l'intentionnalité chorégraphique de l'auteur, le chercheur réactive aujourd'hui la source dans une volonté de connaître et reconstituer les pratiques dansées du XVII ${ }^{\mathrm{e}}$ siècle. 


\section{Bianca Maurmayr}

Bianca Maurmayr est maîtresse de conférences à l'université de Lille, département Arts-Danse, depuis septembre 2020. Ses principaux domaines de recherche concernent I'histoire de la danse et du corps à l'époque moderne, les transferts culturels et l'articulation entre théorie et pratique des arts. Elle fait partie du bureau de l'association des Chercheurs en danse (ACD) et est membre d'AIRDanza.

\section{NOTES}

La publication de cette contribution a reçu le soutien de la bourse d'étude llaria Caccia.

1. Sydney Anglo, L'escrime, la danse et l'art de la guerre. Le livre et la représentation du mouvement, Paris, Bibliothèque nationale de France, 2011, p. 8.

2. Sharon Fermor, "On the Question of Pictorial "Evidence" for Fifteenth-Century Dance Technique", dans Dance Research, vol. 5, n 2, 1987, p. 18-32.

3. Judy van Zile, "Do Artists' Renderings Reveal or Conceal ? Images of Dance in Korea ", dans Barbara Sparti, Judy van Zile (dir.), Imaging Dance. Visual Representations of Dancers and Dancing, Hidelsheim/ Zürich/New York, Georg Olms Verlag, 2011, p. 35-52.

4. Versions consultées: BNF, Livres rares, Rés-V-2566 ; INHA, collections Jacques Doucet, NUM 12 RES 333, disponible en ligne : https://bibliotheque-numerique. inha.fr/idurl/1/15560.

5. Sara Mamone, "Arte e spettacolo. La partita senza fine ", dans Jérôme de La Gorce (dir.), Iconographie et arts du spectacle, actes du séminaire (Paris, 1992), Langres, Klincksieck, 1996, p. 67. Nous traduisons. La chercheuse utilise le concept de monument / document tel qu'il a été formulé par Jérôme de La Gorce (s. v. " Documento/Monumento ", Enciclopedia Einaudi, Turin, Einaudi, 1978, vol. v, p. 38-43).

6. Ibid. : « Si potrebbe stabilire una sorta di scala dell'intenzionalità spettacolare, cioè determinare caso per caso quanto il testimone intenda trasmettere un documento di spettacolo che come tale venga immediatamente percepito o quanto questa intenzionalità si stemperi in intenzionalità di diverso genere. » Nous traduisons.

7. À propos de ce transfert culturel, voir Mickaël Bouffard, Christian Schirm, Jean-Michel Vinciguerra (dir.), Un air d'Italie. L'Opéra de Paris de Louis XIV à la Révolution, cat. exp. (Paris, Opéra, 2019), Paris, Bibliothèque nationale de France/Réunion des musées nationaux, 2019 ; Bianca Maurmayr, Une culture chorégraphique au fil des airs. Transferts et adaptations poḯtiques entre Venise et Paris au XVII' siècle, thèse, université Côte d'Azur, 2018.

8. Issus du même milieu géographique, ils adhérent ensemble à de multiples cercles culturels de la ville de Florence - dont l'Accademia degli apatisti - et sont responsables de la représentation graphique de plusieurs occasions festives et spectaculaires, dont Le Nozze degli dei, mais aussi le Carosello Notturno pour Della Bella (1637) et l'Hipermestra pour Spada (1658). Ils sont alors très proches du monde des arts vivants et côtoient à plusieurs reprises les artistes issus du domaine du théâtre et de la danse. Notons par ailleurs qu'une série de douze essais sur les dix-huit planches gravées est conservée à la bibliothèque Marucelliana de Florence, référée peut-être à la représentation florentine de $L a$ Finta Pazza (I-Fm, Incisioni XXXIV, nn. 42-53); aussi le volume est réédité par Balbi en 1649 lors de son séjour à Bruxelles (B-Ba, Fonds iconographique, Album XI/1).

9. Les planches conservées à l'INHA sont par ailleurs naïvement colorées à la gouache, on suppose par Louis XIV lui-même, ce qui confirmerait le fait que l'exemplaire ait appartenu aux collections de la famille royale. Le dessin d'enfant ou le gribouillis qui ouvre l'exemplaire est particulièrement touchant.

10. Voir Annie Charon, "Les livres de fête en France (XVII $-\mathrm{XVIII}$ siècles) ", dans Dominique Morelon (dir.), Chroniques de l'éphémère. Le livre de fête dans la collection Jacques Doucet, Paris, Publications de l'Institut national d'histoire de l'art, 2010.

11. Benoît Bolduc, "Stefano Della Bella, inventeur des gravures des Nozze degli dei (1637) et de Mirame (1641) », dans Marc Bayard (dir.), Rome-Paris, 1640. Transferts culture/s et renaissance d'un centre artistique, Rome/ Paris, Académie de France à Rome/Somogy Éditions d'Art, 2010, p. 482.

12. Voir Aby Warburg, La Naissance de Vénus et Le Printemps de Sandro Botticelli. Étude des représentations de l'Antiquité dans la première Renaissance italienne, Paris, Éditions Allia, 2007 [éd. orig. : Sandro Botticellis Geburt der Venus und Frühling : eine Untersuchung über die Vorstellungen von der Antike in den italienischen Frührenaissance, Hambourg, Leopold Voss, 1893] ; Mathieu Bouvier, "Le rituel du serpent, Warburg ", dans www.pourunatlasdesfigures.net, Mathieu Bouvier (dir.), Lausanne (HES-SO), La Manufacture, 2018 (consulté le 6 avril 2020).

13. Caroline Joubert, "Avant-propos », dans Eadem (dir.), Stefano Della Bella 1610-1664, Paris, Éditions de la Réunion des musées nationaux/Musée des BeauxArts de Caen, 1998, p. 9. On reconnaît cette souplesse et flexibilité dans ses Divers Exercices de cavalerie ou dans la suite des Cinq Morts.

14. Les cinq gravures des décors de la pièce sont présentées dans l'argument traduit en français, intitulé L'Explication des décorations du théâtre, et les Arguments de la pièce, puis publiées dans l'ouvrage bilingue Scene e machine preparate alle nozze di Teti/Décorations et machines aprestées aux Nopces de Tétis, édité presque dix ans après la mise en scène de La Finta Pazza, à l'occasion de la représentation des Noces de Pélée et de Thétis (1654).

15. Philippe Beaussant, Le Ballet des singes et des autruches, Paris, Gallimard-Le Promeneur/INHA, 2010, p. 10.

16. De même qu'« intentionnalité chorégraphique ", nous créons cette expression d'après celle, de Mamone, de «non-préméditation spectaculaire ». Voir Mamone, 1996, cité n. 5, p. 68. 
17. Guillemette Bolens, Le Style des gestes. Corporéité et kinésie dans le récit littéraire, Genève, BHMS, 2008, p. 3-4.

18. Susan Leigh Foster, "Chorégraphier I'histoire", traduit par Marian del Valle, dans Marian del Valle, Bianca Maurmayr, Marina Nordera, Camille Paillet, Alessandra Sini (dir.), Pratiques de la pensée en danse. Les Ateliers de la danse, Paris, L'Harmattan (collection «Thyrse »), 2020, p. 173-179.

19. Je tiens à remercier Sigfried T'Hofft, que j'ai eu l'honneur de rencontrer à Rothenfels en 2016, pour cette importante suggestion d'interprétation.

20. Beaussant, 2010, cité n. 15, p. 25.

21. Je tiens à remercier Catherine Turocy pour la disponibilité et la générosité qu'elle m’a accordées. Pour visualiser le costume, voir http://janestein-stage-elements. com/gallery.html

22. Nous amplifions la valeur sémantique du mot « texte » à toute unité documentaire qui recèle une signification historique et détermine une interaction entre un émetteur (l'auteur ou le graveur) et un destinataire (l'observateur, historique ou contemporain). Voir Liliane Louvel, Texte/Image, Image à lire, texte à voir, Rennes, Presses universitaires de Rennes, 2002, p. 147-222.

23. À noter qu'un mécanisme inséré à l'intérieur du costume permettait d'allonger, de raccourcir, peut-être même d'incliner le cou des oiseaux, leur donnant ainsi un effet de présence. Au sujet de I'anthropologie du geste en relation au costume, voir Marina Nordera, "Les accessoires comme prolongement du corps en scène ", dans Roxane Martin, Marina Nordera (dir.), Les arts de la scène à l'épreuve de l'histoire : les objets et les méthodes de l'historiographie des spectacles produits sur la scène française, 1635-1906, actes de colloque (Nice, 2009), Paris, H. Champion, 2009, p. 53-64.

24. La sensation de lourdeur est renforcée par le noir du lavis ; la matière légère des aquarelles de la version conservée à l'INHA allège les figures et nuance finement les ombres du corps.

25. Collection Michel Hennin. Estampes relatives à I'Histoire de France, Bibliothèque nationale de France, département Estampes et Photographie, RESERVE FOLQB-201 (58). (Hennin, 5174).

26. Dóra Kiss, Saisir le mouvement. Écrire et lire les sources de la belle danse (1700-1797), Paris, Classiques Garnier, 2016, p. 371-458.

27. Je remercie Petra Dotlačilová pour son aide précieuse dans l'identification de ce costume. Sur la relation entre technique dansée, représentation gravée et costume, voir Mickaël Bouffard, Jérôme de La Gorce, "The Convergence of Dancing and Drawing Practices in the Reign of Louis XIV: Costume Designes from the Edmond de Rothschild Collection in the Louvre ", dans Dance Research, vol. 34, n 1, 2016, p. 1-29.

28. Notons que, pour renforcer cette allure militaire, les danseurs sont représentés dans des positions ouvertes, parfois avec un genou plié, provoquant un transfert du poids du corps sur la jambe concernée, très ancrés dans le sol. Della Bella utilise cette même convention, accouplée à un plus grand dynamisme, dans une de ses gravures pour Le Nozze degli dei ("Scena Grotta di Vulcano »), où les adeptes de Vulcain forgent leurs armes et battent rythmiquement leurs marteaux sur l'enclume jusqu'à ce qu'ils affrontent les partisans de Mars.

29. Voir Jennifer Nevile, Footprints of the Dance: An Early Seventeenth-Century Dance Master's Notebook, Leiden/Boston, Brill, 2018.

30. Sur ces danses à caractère militaire et spectaculaire, voir Barbara Sparti, «Moresca and Mattaccino. Where are the Spanish Antecedents? Where are the Moors? ", dans Alessandro Pontremoli, Patrizia Veroli (dir.), Passi, tracce, percorsi. Scritti sulla danza italiana in omaggio a José Sasportes, Rome, Aracne, 2012, p. 19-22; Charlotte Gschwandtner, Moresca. Vielfalt und Konstanten einer Tanzpraxis zwischen 15. und frühen 17. Jahrhundert, Leipzig, Leipziger Univeristätsverlag $\mathrm{GmbH}, 2017$. 